\title{
ON THE OBLIQUE WAVE PENETRATION IN ELASTIC SOLIDS WITH A DOUBLY PERIODIC ARRAY OF CRACKS
}

\author{
BY \\ EDOARDO SCARPETTA (Dipartimento di Ingegneria dell'Informazione e Matematica Applicata, \\ Universita' di Salerno, 84084 Fisciano (SA), Italy) \\ AND
}

MEZHLUM A. SUMBATYAN (Research Institute of Mechanics and Applied Mathematics, 200/1 Stachki Prospect, Rostov-on-Don 344090, Russia)

\begin{abstract}
In the context of wave propagation in damaged (elastic) solids, an analytical approach for oblique penetration of a plane wave through a doubly periodic array of cracks is developed. By using a uniform approximation in one-mode range previously obtained, we give explicit representations for the wave field throughout the structure and the relevant parameters. Two figures show the peculiarity of such results.
\end{abstract}

1. Introduction. Wave propagation through a medium with distributed discontinuities is a concern in many practical problems regarding, for example, composite materials, protection from earthquakes, or ultrasonic methods for nondestructive testing.

There are principally two different approaches to this subject. The first one considers randomly distributed discontinuities, and a brief survey of the works devoted to this direction of research is given in [1]. The second approach assumes well-organized structures, in which the discontinuities are distributed in a regular periodic manner (typically, in a two-dimensional configuration).

An exact numerical procedure to solve this type of so-called doubly-periodic problem was first applied by Achenbach and Li [2] and by Angel and Achenbach [3]. On the contrary, in our recent paper [4], we have proposed a new analytical method which leads to explicit results for one-mode penetration into a doubly-periodic lattice, in the normal incidence case. Assuming an arbitrary (finite) number of vertical arrays, it is shown there that the wave properties of such well-organized structures are considerably different from those typical of structures with randomly distributed discontinuities.

In the present paper, we aim to extend this method to the oblique incidence case, namely, to the case in which penetration occurs with an arbitrary angle of incidence. 
In this connection, we shall use some of our analytical results established in [5] for the single array of discontinuities.

The paper is especially addressed to wave propagation in an elastic context, so that the (scalar) wave field and the discontinuities of concern will be interpreted as anti-plane displacement and cracks in a solid medium, respectively. However, the mathematical framework can also be applied for similar problems in acoustics or in electromagnetism.

\section{Formulation of the problem and reduction to a linear algebraic system.} The formulation is quite classical [2], [4]. We consider a plane scalar wave penetrating in an elastic medium in which there is a doubly-periodic lattice. This consists of $M(\geq 2)$ identical planes, vertically located at $x=0, d, 2 d, \ldots,(M-1) d$, each of them containing an infinite periodic array of co-planar cracks. The period of a vertical array is $2 a$, the opening between two neighbouring cracks is $2 b$ (around $|y|=0,2 a, 4 a, \ldots$ ), the distance between the cracked planes is $d$ (see Fig. 1). We assume that the time dependence is of harmonic type, so that the wave field $\psi(x, y)$ satisfies throughout the Helmholtz equation

$$
\left(\partial_{x x}+\partial_{y y}\right) \psi+\kappa^{2} \psi=0
$$

where $\kappa=\omega / c$ is the wave number, $\omega$ the angular frequency, and $c$ the (transverse) wave speed. Of course, $\psi(x, y) e^{-i \omega t}$ gives $u_{z}(x, y, t)$, the only nontrivial component of the displacement vector $\left(i^{2}=-1\right)$.

The incident wave, of unitary amplitude, has the form

$$
\psi_{0}=e^{i \kappa(x \cos \vartheta+y \sin \vartheta)},
$$

$\vartheta$ being the angle of incidence (cf. [5], [6]).

The natural symmetry and periodicity of the problem suggest that $\psi$ can be written as

$$
\psi(x, y)=e^{i \kappa y \sin \vartheta} \varphi(x, y)
$$

where $\varphi$ is a periodic function with respect to $y: \varphi(x, y)=\varphi(x, y+2 a) \forall x, y[6]$. By the same token, the geometrical context of the problem can be restricted to a single layer $|y|<a$ with openings $|y|<b$, and the wave field $\varphi$ can be represented in the following forms along the various regions:

$$
\begin{aligned}
& \varphi_{l}= e^{i \kappa x \cos \vartheta}+R e^{-i \kappa x \cos \vartheta}+\sum_{n \neq 0} A_{n} e^{\left(\tau_{n} x-i \pi n y / a\right)}, \quad x<0, \\
& \varphi_{m}= B_{0}^{m} \cos \{\kappa[x-(m-1) d] \cos \vartheta\}+C_{0}^{m} \cos [\kappa(x-m d) \cos \vartheta] \\
&+\sum_{n \neq 0}\left\{B_{n}^{m} \operatorname{ch}\left[\tau_{n}[x-(m-1) d]\right]+C_{n}^{m} \operatorname{ch}\left[\tau_{n}(x-m d)\right]\right\} e^{-i \pi n y / a}, \\
&(m-1) d<x<m d, \quad m=1,2, \ldots, M-1 ;
\end{aligned}
$$




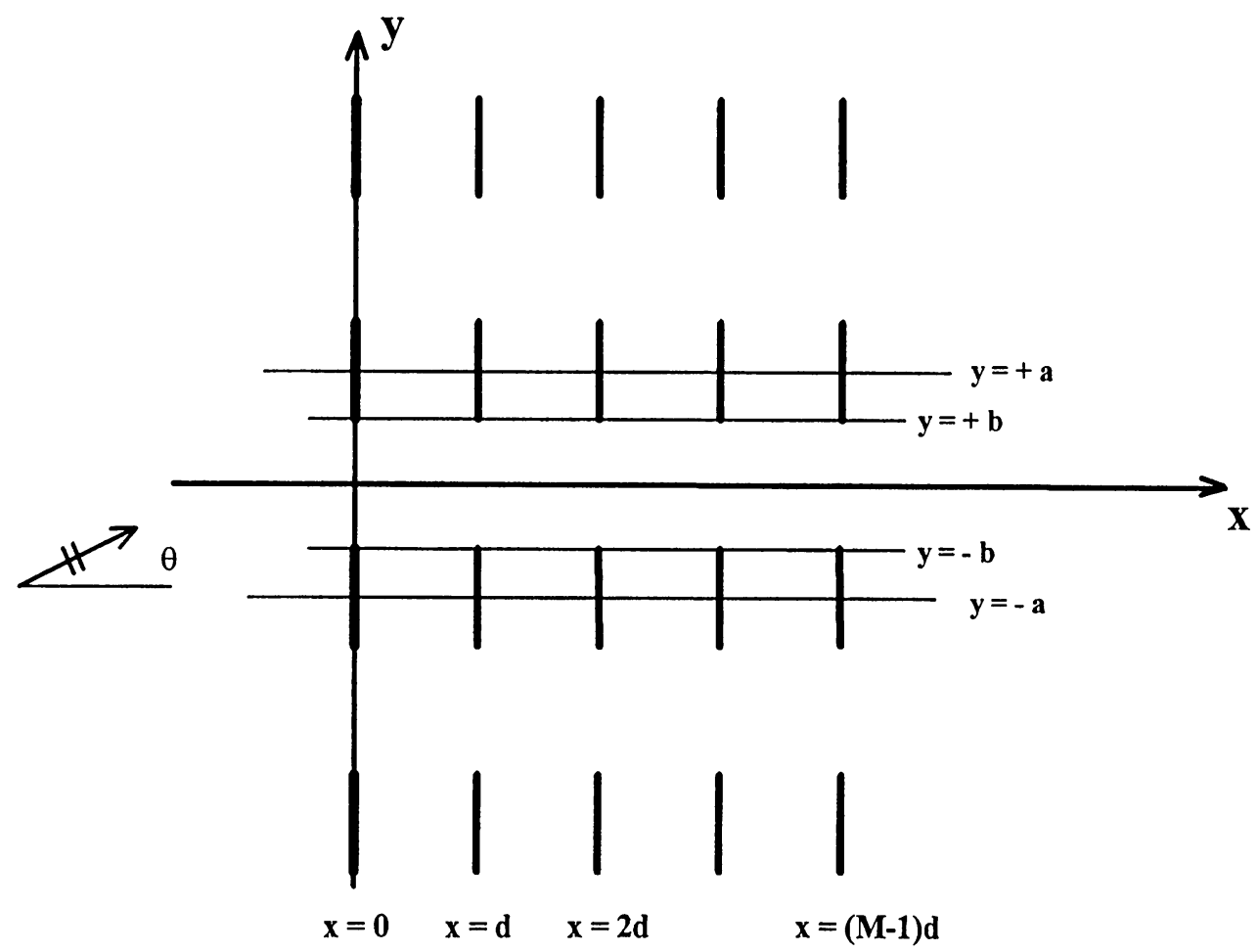

FIG. 1. Oblique penetration of a plane incident wave through a doubly-periodic array of cracks

$$
\varphi_{r}=T e^{i \kappa[x-(M-1) d] \cos \vartheta}+\sum_{n \neq 0} D_{n} e^{-\tau_{n}[x-(M-1) d]-i \pi n y / a}, \quad x>(M-1) d
$$

where

$$
\tau_{n}=\left[(\pi n / a-\kappa \sin \vartheta)^{2}-\kappa^{2}\right]^{1 / 2}
$$

in order that each term of such equations, after being multiplied by $e^{i \kappa y \sin \vartheta}$, be a trivial solution of Eq. (2.1).

In the above formulas, all capital letters denote unknown constants, ch (or sh) stands for hyperbolic cosine (or sine), and the symbol $\sum_{n \neq 0}$ means summation $\forall n \in\{\ldots,-2$, $-1,1,2, \ldots\}$. We prefer to let explicitly appear the terms with $n=0$; note this in particular for the constants $R \equiv A_{0}$ in (2.4a) and $T \equiv D_{0}$ in (2.4c).

The natural traction-free boundary condition on the cracks' faces requires that $\partial \varphi / \partial x$ $=0$ for $b<|y|<a$ and $x=(m-1) d, m=1, \ldots, M$. Assuming also a continuity property of $\partial \varphi / \partial x$ through the openings, i.e., for $|y|<b$ and $x=(m-1) d, m=1, \ldots, M$, we can introduce some new unknown functions $g_{m}(y)$, related to the stress components along 
the openings, as follows (cf. [4]):

$$
\begin{aligned}
& \frac{\partial \varphi_{l}}{\partial x}=\frac{\partial \varphi_{1}}{\partial x}=\left\{\begin{array}{ll}
g_{1}(y), & |y|<b, \\
0, & b<|y|<a,
\end{array} \quad x=0 ;\right.
\end{aligned}
$$

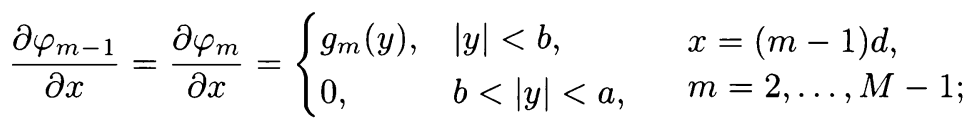

$$
\begin{aligned}
& \frac{\partial \varphi_{M-1}}{\partial x}=\frac{\partial \varphi_{r}}{\partial x}=\left\{\begin{array}{ll}
g_{M}(y), & |y|<b, \\
0, & b<|y|<a,
\end{array} \quad x=(M-1) d .\right.
\end{aligned}
$$

By integration of the above equations over $|y|<a$, we easily get

$$
\begin{aligned}
i \kappa(1-R) \cos \vartheta & =\frac{1}{2 a} \int_{-b}^{b} g_{1}(t) d t=\kappa \cos \vartheta C_{0}^{1} \sin (\kappa d \cos \vartheta) \\
\kappa \cos \vartheta C_{0}^{m} \sin (\kappa d \cos \vartheta) & =\frac{1}{2 a} \int_{-b}^{b} g_{m}(t) d t \\
& =-\kappa \cos \vartheta B_{0}^{m-1} \sin (\kappa d \cos \vartheta), \quad m=2, \ldots, M-1, \\
i \kappa T \cos \vartheta & =\frac{1}{2 a} \int_{-b}^{b} g_{M}(t) d t=-\kappa \cos \vartheta B_{0}^{M-1} \sin (\kappa d \cos \vartheta) .
\end{aligned}
$$

Repeating the integration after multiplication by $e^{i \pi n^{\prime} y / a}, n^{\prime}= \pm 1, \pm 2, \ldots$, and considering that

$$
\int_{-a}^{a} e^{i \pi\left(n^{\prime}-n\right) y / a} d y=2 a \delta_{n, n^{\prime}}
$$

we also get:

$$
\begin{gathered}
\tau_{n} A_{n}=\frac{1}{2 a} \int_{-b}^{b} g_{1}(t) e^{i \pi n t / a} d t=-\tau_{n} C_{n}^{1} \operatorname{sh}\left(\tau_{n} d\right), \\
-\tau_{n} C_{n}^{m} \operatorname{sh}\left(\tau_{n} d\right)=\frac{1}{2 a} \int_{-b}^{b} g_{m}(t) e^{i \pi n t / a} d t=\tau_{n} B_{n}^{m-1} \operatorname{sh}\left(\tau_{n} d\right), \\
m=2,3, \ldots, M-1, \\
-\tau_{n} D_{n}=\frac{1}{2 a} \int_{-b}^{b} g_{M}(t) e^{i \pi n t / a} d t=\tau_{n} B_{n}^{M-1} \operatorname{sh}\left(\tau_{n} d\right) .
\end{gathered}
$$

Equations $(2.7,2.8)$ give all the unknown constants in terms of the functions $g_{m}$.

A continuity property is of course assumed also for the wave fields through the openings:

$$
\begin{aligned}
\varphi_{l} & =\varphi_{1} & & \text { at } x=0, \\
\varphi_{m} & =\varphi_{m-1} & & \text { at } x=(m-1) d, \\
\varphi_{M-1} & =\varphi_{r} & & \text { at } x=(M-1) d,
\end{aligned}
$$


for $|y|<b$, so that the following equations are implied from (2.4):

$$
\begin{aligned}
& 1+R+\sum_{n \neq 0} A_{n} e^{-i \pi n y / a} \\
& =B_{0}^{1}+C_{0}^{1} \cos (\kappa d \cos \vartheta)+\sum_{n \neq 0}\left[B_{n}^{1}+C_{n}^{1} \operatorname{ch}\left(\tau_{n} d\right)\right] e^{-i \pi n y / a} \\
& B_{0}^{m}+C_{0}^{m} \cos (\kappa d \cos \vartheta)+\sum_{n \neq 0}\left[B_{n}^{m}+C_{n}^{m} \operatorname{ch}\left(\tau_{n} d\right)\right] e^{-i \pi n y / a} \\
& =B_{0}^{m-1} \cos (\kappa d \cos \vartheta)+C_{0}^{m-1}+\sum_{n \neq 0}\left[B_{n}^{m-1} \operatorname{ch}\left(\tau_{n} d\right)+C_{n}^{m-1}\right] e^{-i \pi n y / a} \\
& \quad m=2,3, \ldots, M-1 \\
& B_{0}^{M-1} \cos (\kappa d \cos \vartheta)+C_{0}^{M-1}+\sum_{n \neq 0}\left[B_{n}^{M-1} \operatorname{ch}\left(\tau_{n} d\right)+C_{n}^{M-1}\right] e^{-i \pi n y / a} \\
& =T+\sum_{n \neq 0} D_{n} e^{-i \pi n y / a} .
\end{aligned}
$$

After substituting the values of the constants, taken from Eqs. $(2.7,2.8)$, into these equalities, the problem is clearly reduced to a system of integral equations for the functions $g_{m}(y)(m=1,2, \ldots, M ;|y|<b)$, that of course could be solved numerically for an arbitrary (finite) number of vertical planes and arbitrary values of the physical and geometrical parameters.

However, in the present paper, we aim to study analytically the properties of the structure, and to this end we accept only the following fundamental assumptions (cf. [4], [5]):

a) $0<\kappa a<\pi /(1+\sin \vartheta)$, for given $\vartheta$;

b) $d / a \gg 1$.

The first assumption implies that $\tau_{n}>0 \forall n \neq 0$, which guarantees the so-called onemode regime: this means that at large distance from the structure only plane waves with the given wave number $\kappa$ can be present, so as to grant to constants $R$ and $T$ their full meaning as reflection and transmission coefficients, respectively. The second assumption implies that the vertical planes are sufficiently distant from each other, with respect to the width of the layer. Both these assumptions allow us to put $\operatorname{ch}\left(\tau_{n} d\right) \approx \operatorname{sh}\left(\tau_{n} d\right) \gg 1$ in Eqs. (2.8) and (2.9), so that the terms $B_{n}^{m}, C_{n}^{m}(m=1, \ldots, M-1)$ in the square brackets of (2.9) become negligible (with respect to the other terms containing $\operatorname{ch}\left(\tau_{n} d\right)$ ).

From a physical standpoint, this approximation implies that all nonhomogeneous (standing) waves rapidly decay when they move from one vertical array to the following one (as is assumed in [2], [3]). Actually, it could be shown that this is a good approximation even when the distance $d$ is of the same order as the width $2 a(d / a \geq 2)$; see [4]. 
So, by substituting the values of the constants, from Eqs. $(2.7,2.8)$, in the approximated version (as said above) of Eqs. (2.9), we can finally lay down the following square system of integral equations for the unknowns $g_{1}, \ldots, g_{M}$ in the interval $|y|<b$ :

$$
\begin{gathered}
\frac{1}{2 a} \int_{-b}^{b} g_{1}(t)\left\{(2 \kappa \cos \vartheta)^{-1}[\operatorname{ctg}(\kappa d \cos \vartheta)-i]-\sum_{n \neq 0}\left(1 / \tau_{n}\right) e^{i \pi n(t-y) / a}\right\} d t \\
-[4 a \kappa \cos \vartheta \sin (\kappa d \cos \vartheta)]^{-1} \int_{-b}^{b} g_{2}(t) d t=1 ; \\
\frac{1}{2 a} \int_{-b}^{b} g_{m}(t)\left\{(\kappa \cos \vartheta)^{-1} \operatorname{ctg}(\kappa d \cos \vartheta)-\sum_{n \neq 0}\left(1 / \tau_{n}\right) e^{i \pi n(t-y) / a}\right\} d t \\
-[4 a \kappa \cos \vartheta \sin (\kappa d \cos \vartheta)]^{-1} \int_{-b}^{b}\left[g_{m-1}(t)+g_{m+1}(t)\right] d t=0 \\
(m=2,3, \ldots, M-1) ; \\
\frac{1}{2 a} \int_{-b}^{b} g_{M}(t)\left\{(2 \kappa \cos \vartheta)^{-1}[\operatorname{ctg}(\kappa d \cos \vartheta)-i]-\sum_{n \neq 0}\left(1 / \tau_{n}\right) e^{i \pi n(t-y) / a}\right\} d t \\
-[4 a \kappa \cos \vartheta \sin (\kappa d \cos \vartheta)]^{-1} \int_{-b}^{b} g_{M-1}(t) d t=0 .
\end{gathered}
$$

Note that, on putting $\vartheta=0$ throughout the above, the formulation of the problem for the normal incidence case, dealt with in [4], is recovered.

As in [5], let us now consider the auxiliary integral equation:

$$
\frac{1}{2 a} \int_{-b}^{b} h(t)\left\{\sum_{n \neq 0}\left(1 / \tau_{n}\right) e^{i \pi n(t-y) / a}\right\} d t=1, \quad|y|<b .
$$

In terms of the new function $h$, we can derive from system (2.10) (by linearity):

$$
\begin{aligned}
& g_{1}(y)=\left\{(4 a \kappa \cos \vartheta)^{-1}[\operatorname{ctg}(\kappa d \cos \vartheta)-i] G_{1}\right. \\
&\left.-[4 a \kappa \cos \vartheta \sin (\kappa d \cos \vartheta)]^{-1} G_{2}-1\right\} h(y) ; \\
& g_{m}(y)=\left\{(2 a \kappa \cos \vartheta)^{-1} \operatorname{ctg}(\kappa d \cos \vartheta) G_{m}\right. \\
&\left.-[4 a \kappa \cos \vartheta \sin (\kappa d \cos \vartheta)]^{-1}\left(G_{m-1}+G_{m+1}\right)\right\} h(y), \\
& m=2, \ldots, M-1 ; \\
& g_{M}(y)=\left\{(4 a \kappa \cos \vartheta)^{-1}[\operatorname{ctg}(\kappa d \cos \vartheta)-i] G_{M}\right. \\
&\left.-[4 a \kappa \cos \vartheta \sin (\kappa d \cos \vartheta)]^{-1} G_{M-1}\right\} h(y) ;
\end{aligned}
$$

where

$$
G_{M}=\int_{-b}^{b} g_{m}(t) d t, \quad m=1,2, \ldots, M
$$


Set also

$$
H=\int_{-b}^{b} h(t) d t .
$$

Integrating the above equations over $|y|<b$ finally yields the following square system of linear algebraic equations in the unknowns $G_{1}, \ldots, G_{M}$ :

$$
\begin{aligned}
& \left\{1-(4 a \kappa \cos \vartheta)^{-1}[\operatorname{ctg}(\kappa d \cos \vartheta)-i] H\right\} G_{1} \\
& \quad+\left\{[4 a \kappa \cos \vartheta \sin (\kappa d \cos \vartheta)]^{-1} H\right\} G_{2}=-H
\end{aligned}
$$

$\left\{[4 a \kappa \cos \vartheta \sin (\kappa d \cos \vartheta)]^{-1} H\right\} G_{m-1}+\left\{1-(2 a \kappa \cos \vartheta)^{-1} \operatorname{ctg}(\kappa d \cos \vartheta) H\right\} G_{m}$

$$
+\left\{[4 a \kappa \cos \vartheta \sin (\kappa d \cos \vartheta)]^{-1} H\right\} G_{m+1}=0, \quad m=2, \ldots, M-1
$$

$\left\{[4 a \kappa \cos \vartheta \sin (\kappa d \cos \vartheta)]^{-1} H\right\} G_{M-1}$

$$
+\left\{1-(4 a \kappa \cos \vartheta)^{-1}[\operatorname{ctg}(\kappa d \cos \vartheta)-i] H\right\} G_{M}=0 .
$$

The constant $H$ appearing in this system is an integral measure of the solution $h$ of Eq. (2.11). A uniformly approximated representation for this function, valid in the one-mode regime, has been obtained in [5]; for its integral, we obtained (see Sec. 5 of [5]):

$$
\begin{aligned}
& H=\left\{-\pi /\left[\left(a_{11} a_{22}+a_{12} a_{21}\right) \ln \xi_{0}\right]\right\}\left(\left\{1-\left[1-\pi /\left(a q_{1}\right)\right] \xi_{0}^{4}\right\} a_{22}\right. \\
&\left.+\left\{1-\left[1-\pi /\left(a \tau_{1}\right)\right] \xi_{0}^{4}\right\} a_{21}\right) \quad(\kappa a<\pi /(1+\sin \vartheta)),
\end{aligned}
$$

where

$$
\begin{aligned}
& \xi_{0}=\sin (\pi b / 2 a) \\
& \eta_{0}=\cos (\pi b / 2 a) \\
& q_{1}=\tau_{-1}=\left[(\pi / a+\kappa \sin \vartheta)^{2}-\kappa^{2}\right]^{1 / 2}
\end{aligned}
$$

and

$$
\begin{aligned}
& a_{11}=1-\left[1-\pi /\left(a q_{1}\right)\right]\left(\xi_{0}^{4}-\eta_{0}^{4} / \ln \xi_{0}\right), \\
& a_{12}=1-\left[1-\pi /\left(a \tau_{1}\right)\right]\left(\xi_{0}^{4}-\eta_{0}^{4} / \ln \xi_{0}\right), \\
& a_{21}=1-\left[1-\pi /\left(a q_{1}\right)\right]\left(1-\eta_{0}^{4}\right), \\
& a_{22}=1-\left[1-\pi /\left(a \tau_{1}\right)\right]\left(1-\eta_{0}^{4}\right) .
\end{aligned}
$$

Note that, despite the fact that the solution $h$ of Eq. (2.11) is generally complex-valued, the constant $H$, in the considered one-mode regime, is actually real.

Of course, once system (2.14) is solved, the wave field can be explicitly derived by inserting in Eqs. (2.4) what is calculated from (2.12) and (2.7, 2.8). In particular, the reflection and transmission coefficients are given by Eqs. (2.7a) and (2.7c) as follows:

$$
R=1-(2 a i \kappa \cos \vartheta)^{-1} G_{1}, \quad T=(2 a i \kappa \cos \vartheta)^{-1} G_{M}
$$


3. Solution of the linear system and main results. System (2.14) appears in the form

$$
\left(\begin{array}{ccccc|c}
p & c & & & f \\
c & \beta & c & & 0 & 0 \\
& \ddots & \ddots & \ddots & & \\
& & c & \beta & c & 0 \\
0 & & & c & p & 0
\end{array}\right)
$$

where

$$
\begin{aligned}
& f=-H \\
& c=[4 a \kappa \cos \vartheta \sin (\kappa d \cos \vartheta)]^{-1} H \\
& \beta=1-(2 a \kappa \cos \vartheta)^{-1} \operatorname{ctg}(\kappa d \cos \vartheta) H \\
& p=1-(4 a \kappa \cos \vartheta)^{-1}[\operatorname{ctg}(\kappa d \cos \vartheta)-i] H
\end{aligned}
$$

Its solution can be obtained by using Cramer's rule as follows:

$$
\begin{aligned}
G_{1} & =\left(f / J_{M}\right)\left(p I_{M-2}-c^{2} I_{M-3}\right), \\
G_{M} & =\left(f / J_{M}\right)(-c)^{M-1}, \\
G_{m} & =\left(f / J_{M}\right)(-c)^{m-1}\left(p I_{M-m-1}-c^{2} I_{M-m-2}\right), \quad m=2, \ldots, M-1,
\end{aligned}
$$

where we have denoted the full determinant of the system by

$$
J_{M}=p^{2} I_{M-2}-2 p c^{2} I_{M-3}+c^{4} I_{M-4}
$$

and the determinant of order $N$ by

$$
I_{N}=\left|\begin{array}{ccccc}
\beta & c & & & \\
c & \beta & c & & 0 \\
& c & \ddots & \ddots & \\
& & \ddots & \beta & c \\
0 & & & c & \beta
\end{array}\right| .
$$

We conventionally assume that $I_{0}=1, I_{-1}=0, I_{-2}=-c^{-2}$.

It is well known [7] that

$$
I_{N}=\beta I_{N-1}-c^{2} I_{N-2}=\left(x_{1}^{N+1}-x_{2}^{N+1}\right) /\left(x_{1}-x_{2}\right)
$$

where $x_{1}$ and $x_{2}$ are the roots of the quadratic equation

$$
x^{2}-\beta x+c^{2}=0
$$

and can be represented as

$$
x_{1}=-r e^{i \alpha}, \quad x_{2}=-r e^{-i \alpha},
$$


where

$$
\begin{aligned}
r & =c=H /[4 a \kappa \cos \vartheta \sin (\kappa d \cos \vartheta)] \\
\cos \alpha & =-\beta /(2 c)=\cos (\kappa d \cos \vartheta)-2(\kappa d \cos \vartheta)(H d / a)^{-1} \sin (\kappa d \cos \vartheta)
\end{aligned}
$$

We note that the argument $\alpha$ can attain real as well as (purely) imaginary values; this of course depends on the given geometrical and physical parameters according to the sign-negative or positive, respectively —of the discriminant $\left(\beta^{2}-4 c^{2}\right)$. Also, the sign of $r=c$ in Eq. $(3.5 \mathrm{~b})_{1}$ is that of $H / \sin (\kappa d \cos \vartheta)$, and this is surely positive for small enough frequencies (we recall from [5, Sec. 6] that, in the limit $\kappa a \rightarrow 0, H \approx \pi /\left(-\ln \xi_{0}\right)+O\left(\kappa^{2} a^{2}\right)$; cf. [4, Sec. 3] for the case of normal incidence, in which the constant $H(\vartheta=0)$ is always positive). Equations (3.5), however, are valid for arbitrary incidence angle and frequency in the one-mode range.

The complete solution of system (2.14) enables us to give explicit expressions for the reflection and transmission coefficients. By Eqs. (2.16), (3.2) and some formal transformations ${ }^{1}$, we finally arrive at the following formulas:

$$
\begin{aligned}
R & =\left(1+\frac{i H}{2 a \kappa \cos \vartheta}\left\{1-e^{i \kappa d \cos \vartheta}[\cos \alpha-\sin \alpha \operatorname{ctg}(M \alpha)]\right\}\right)^{-1} \\
T & =\frac{i H}{2 a \kappa \cos \vartheta}[\sin \alpha / \sin (M \alpha)] R .
\end{aligned}
$$

As can be proved, these equations guarantee that the well-known property of the balance of rates of energies

$$
|R|^{2}+|T|^{2}=1 \quad \text { (for one-mode case) }
$$

identically holds, independently of the value of the constant $H$.

4. Analysis of the wave properties of the structure. As is evident from Eqs. $(3.6,3.7)$, the dependence of the parameters $R$ and $T$ on the number $M$ of vertical arrays, which in some sense represents the distance through the structure, is only determined by the value of the parameter $\alpha$. As follows from Eq. $(3.5 \mathrm{~b})_{2}$, for small values of the product $\kappa d \cos \vartheta, \alpha$ is real and given approximately as

$$
\alpha \approx\left[1+4(H d / a)^{-1}\right]^{1 / 2}(\kappa d \cos \vartheta) .
$$

So, in this case, the reflection and transmission coefficients possess a periodic behaviour with respect to $M$.

When the product $\kappa d \cos \vartheta$ increases, $\alpha$ can become imaginary, and this implies an exponential decay for $T$ (amounting to a rapid wave attenuation) both with respect to the frequency and with respect to $M$ (i.e., the distance). Such a property evidently follows from Eqs. $(3.6,3.7)$, since $\sin \gamma=i \operatorname{sh} \tilde{\gamma}$ and $\cos \gamma=\operatorname{ch} \tilde{\gamma}$ when $\gamma(=\alpha$ or $M \alpha)$ is imaginary: $\gamma=i \tilde{\gamma}, \tilde{\gamma} \in \mathbb{R}$.

\footnotetext{
${ }^{1}$ For more details about these, see [4, Sec. 4].
} 


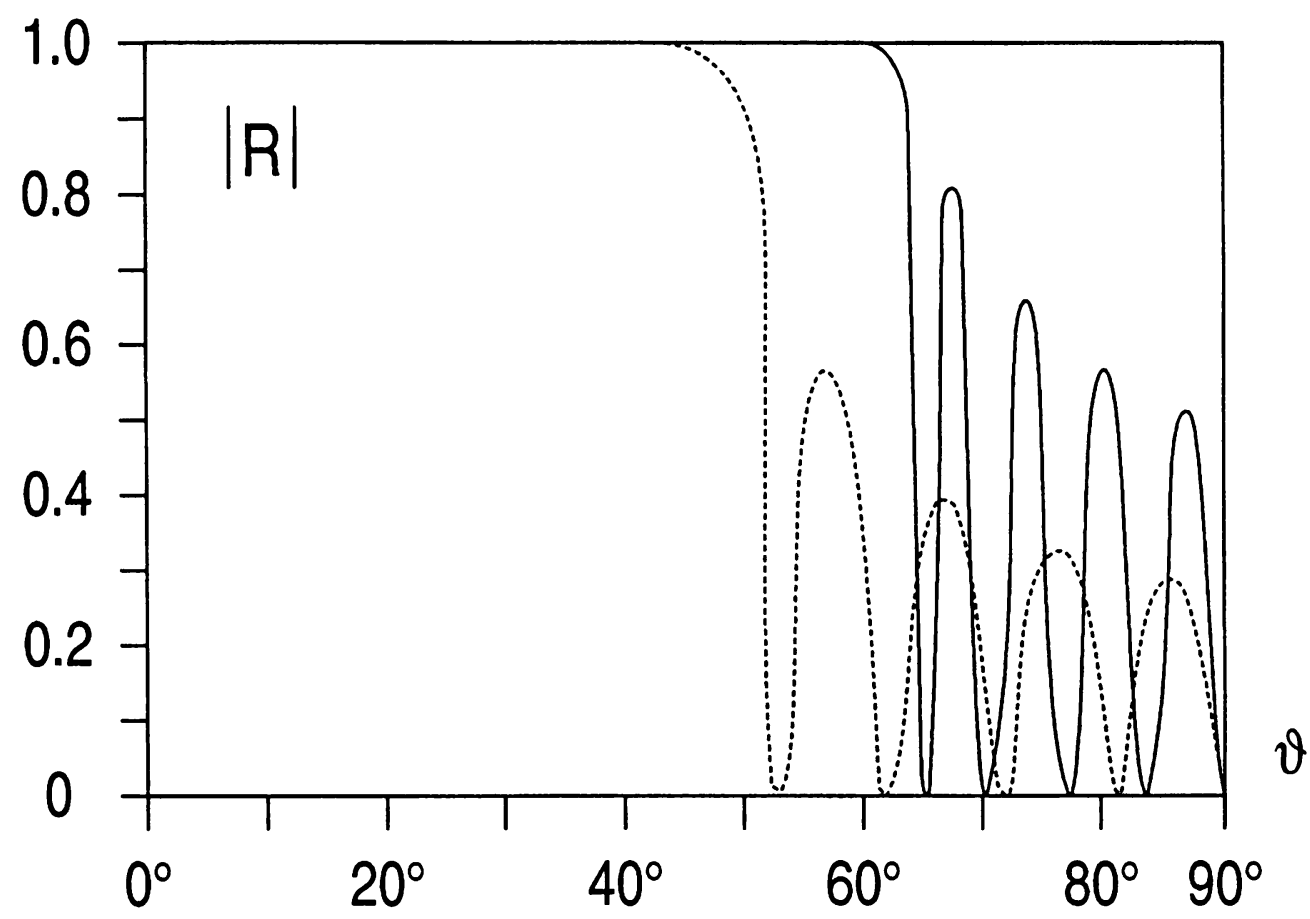

FIG. 2. Dependence of the reflection coefficient $|R|$ upon the angle of incidence $\vartheta(d / a=2, M=5, \kappa d=3)$. Full line: $b / a=0.1$; dotted line: $b / a=0.3$

Two figures show this interesting feature (both of them for two values of the relative opening $b / a$ ). In Fig. 2 the reflection coefficient is given as a function of the angle of incidence (at fixed frequency). The frequency parameter is taken so large $(\kappa d=2 \kappa a=3)$ that, in the case of normal incidence $\left(\vartheta=0^{\circ}\right)$, even for a relatively small number $M=5$, the structure does not permit transmission of the wave $(|R| \approx 1$, so that $|T| \approx 0$ due to Eq. (3.8)). When the angle of incidence $\vartheta$ becomes sufficiently large (and so $\cos \vartheta$ small enough), the behaviour of the structure changes from attenuating to oscillating, according to the (real) value of $\alpha$ from Eq. (4.1).

Further, Fig. 3 gives the transmission coefficient as a function of the frequency parameter $\kappa a$ (at fixed $\vartheta=30^{\circ}$ ), and we can see how such a coefficient starts to decay exponentially when $\kappa a$ exceeds certain critical values, namely, when the parameter $\alpha$ becomes imaginary. This figure can be compared with Fig. 5 of [4], which displays the same function $|T|$ vs. $\kappa a$ (with the same values of the relevant geometrical parameters) in the case of normal incidence. It should be noted that an oblique incidence actually enlarges the range of frequency for periodicity with a good transmission (cf. [6]).

Therefore, there are two different ranges of physical and geometrical parameters to which correspond quite different physical properties. This fact distinguishes the wave 


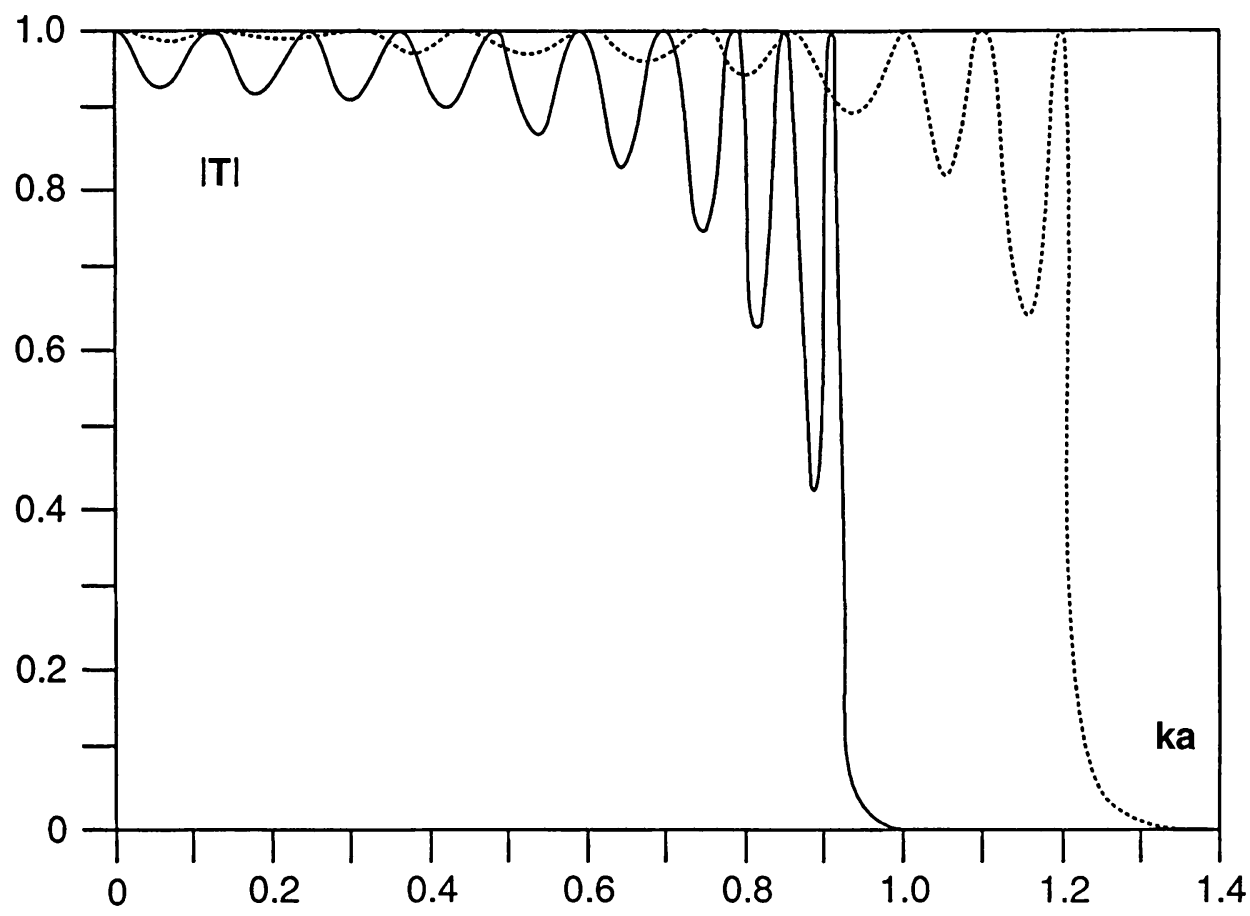

FIG. 3. Dependence of the transmission coefficient $|T|$ upon the frequency parameter $\kappa a\left(d / a=2, M=10, \vartheta=30^{\circ}\right)$. Full line: $b / a=0.1 ;$ dotted line: $b / a=0.3$

properties of well-organized structures from those of randomly distributed structures, in which the wave attenuation always increases with the distance, even for very small values of the frequency [1]. We recall that, in the present context, the transition from the range of periodicity to the range of decay is only determined by the sign of $\left(\beta^{2}-4 c^{2}\right)$, being actually independent on the number $M$ of vertical arrays.

Acknowledgments. The research described in this paper was performed during a visit of Prof. Sumbatyan at the University of Salerno (c/o Dip. Ing. Inf. Mat. Appl.). Financial support for this from G.N.F.M. of the Italian Research Council (C.N.R.) is gratefully acknowledged.

\section{REFERENCES}

[1] Ch. Zhang and D. Gross, Wave attenuation and dispersion in randomly cracked solids. I, II, Internat. J. Engrg. Sci. 31, 841-872 (1993)

[2] J. D. Achenbach and Z. L. Li, Propagation of horizontally polarized transverse waves in a solid with a periodic distribution of cracks, Wave Motion 8, 371-379 (1986)

[3] Y. C. Angel and J. D. Achenbach, Harmonic waves in an elastic solid containing a doubly periodic array of cracks, Wave Motion 9, 377-385 (1987)

[4] E. Scarpetta and M. A. Sumbatyan, On wave propagation in elastic solids with a doubly periodic array of cracks, Wave Motion 25, 61-72 (1997) 
[5] E. Scarpetta and M. A. Sumbatyan, Explicit analytical results for one-mode oblique penetration into a periodic array of screens, IMA Journal of Applied Mathematics 56, 109-120 (1996)

[6] J. D. Achenbach and Z. L. Li, Reflection and transmission of scalar waves by a periodic array of screens, Wave Motion 8, 225-234 (1986)

[7] F. Bowman, An Introduction to Determinants and Matrices, English University Press, London, 1962 